\title{
Constraining The Symmetry Energy (Far) Above Saturation Density Using Elliptic Flow
}

\author{
M.D. Cozma ${ }^{1, \star}$ \\ ${ }^{1}$ IFIN-HH, Reactorului 30, 077125 Măgurele-Bucharest, Romania
}

\begin{abstract}
A QMD transport model that employs a modified momentum dependent interaction (MDI2) potential, supplemented by a phase-space coalescence model fitted to experimental multiplicities of free nucleons and light clusters, is used to study the density dependence of the symmetry energy above the saturation point by a comparison with experimental elliptic flow ratios measured by the FOPI-LAND and ASYEOS collaborations for ${ }^{197} \mathrm{Au}+{ }^{197} \mathrm{Au}$ collisions at $400 \mathrm{MeV} /$ nucleon impact energy. Comparing theoretical predictions with experimental data for neutron-to-proton and neutron-tocharged particles elliptic flow ratios the following constraint is extracted for the slope $L$ and curvature $K_{\text {sym }}$ of symmetry energy at saturation: $L=59 \pm 24$ (exp) \pm 16 (th) \pm 10 (sys) $\mathrm{MeV}$ and $K_{\text {sym }}=0 \pm 370$ (exp) \pm 220 (th) \pm 150 (sys) MeV. Theoretical errors are the result of poorer known model ingredients. Systematical uncertainties are generated by the inability of the transport model to reproduce experimental light-cluster-to-proton multiplicity ratios. A more accurate value for $L$, free of systematical theoretical uncertainties, can be extracted from the neutron-to-proton elliptic flow ratio alone: $L=63 \pm 18(\exp ) \pm 14$ (th) $\mathrm{MeV}$.
\end{abstract}

\section{Introduction}

Knowledge of the density dependence of the isospin dependent part of the equation of state (asy-EoS) of nuclear matter, commonly known as the symmetry energy (SE), is crucial for the understanding of the structure of rare isotopes, dynamics of heavy-ion collisions and properties of astrophysical objects such as neutron stars [1,2]. Experimental studies of isospin diffusion, pygmy and giant dipole resonances, neutron skin thickness and other phenomena has made possible the extraction of constraints with satisfactory accuracy for the density dependence of the SE in the vicinity or below the saturation density $\left(\rho_{0}\right)$ [3]. Similarly, recent advances in theoretical many-body simulations of nuclear matter have allowed increasingly more accurate predictions for the asy-EoS up to densities close to the saturation point [4]. The density dependence far above saturation, $\rho \geq 2 \rho_{0}$, has remained up to now extremely uncertain. Its knowledge is mandatory for a proper understanding of properties of neutron stars such as radius and maximum allowed mass and may provide the key for a simple solution of the so called hyperon puzzle [5].

Intermediate energy heavy-ion collisions (HIC) provide the unique opportunity to study, in terrestrial laboratories, nuclear matter in the vicinity of twice saturation density [6]. To this end, elliptic

^e-mail: dan.cozma@theory.nipne.ro 
flow ratios (EFR) of isospin partners have been shown to be sensitive to the density dependence of SE [7, 8]. Consequently, constraints for the asy-EoS stiffness have been extracted [7, 9-11] from a comparison of theoretical models with experimental data of the FOPI-LAND [12] and ASYEOS [9] collaborations. Due to the fact the neutron-to-proton (npEFR) and neutron-to-hydrogen (nhEFR) elliptic flow ratios probe on average different density regimes, 1.4-1.5 $\rho_{0}$ and 1.0-1.1 $\rho_{0}$ respectively [9], it is in principle possible to extract constraints for both the slope $L$ and curvature $K_{\text {sym }}$ of SE at saturation rather than an average value of the slope at an undetermined density.

The study of Ref. [13] had as main goal the extraction of constraints for the slope $L$ and curvature $K_{\text {sym }}$ from the FOPI-LAND npEFR and ASYEOS neutron-to-charged-particles elliptic flow ratio (nchEFR) experimental data. In this conference proceeding the main modifications to the transport model are briefly presented, supplemented by the main results of that study.

\section{The model}

Heavy-ion collision dynamics is simulated using an upgraded version $[14,15]$ of the Tübingen quantum molecular dynamics model (QMD) transport model [16, 17]. To allow for independent variations of the $L$ and $K_{\text {sym }}$ parameters, the potential component of the MDI Gogny-inspired version of the equation of state of nuclear matter [18] has been modified (and named MDI2) by the addition of a density dependent, momentum independent term proportional to a parameter denoted $D$

$$
\begin{aligned}
\frac{E}{N}(\rho, \beta)= & A_{u}(x, y) \frac{\rho\left(1-\beta^{2}\right)}{4 \rho_{0}}+A_{l}(x, y) \frac{\rho\left(1+\beta^{2}\right)}{4 \rho_{0}}+\frac{B}{\sigma+1} \frac{\rho^{\sigma}}{\rho_{0}^{\sigma}}\left(1-x \beta^{2}\right) \\
& +\frac{D}{3} \frac{\rho^{2}}{\rho_{0}^{2}}\left(1-y \beta^{2}\right)+\frac{1}{\rho \rho_{0}} \sum_{\tau, \tau^{\prime}} C_{\tau \tau^{\prime}} \iint d^{3} \vec{p} d^{3} \vec{p}^{\prime} \frac{f_{\tau}(\vec{r}, \vec{p}) f_{\tau^{\prime}}\left(\vec{r}, \vec{p}^{\prime}\right)}{1+\left(\vec{p}-\vec{p}^{\prime}\right)^{2} / \Lambda^{2}}
\end{aligned}
$$

In the above expression $\rho, \beta$ and $p$ denote the density, isospin asymmetry and momentum variables respectively. The parameters $x$ and $y$ have been introduced to allow independent adjustments of the stiffness parameters $L$ and $K_{\text {sym }}$ of symmetry energy. The free parameters of the equation of state are determined by using the following data as input: value of the optical potential at infinite momentum, effective nucleon mass in isospin symmetric matter, isovector effective mass difference, value of the saturation density of isospin symmetric matter, value of the binding energy at saturation, compressibility $K_{0}$ and skewness $Q_{0}$ parameters of isospin symmetric matter, value of the symmetry energy at a particular density $\tilde{\rho}$ and the slope $L$ and curvature $K_{\text {sym }}$ of symmetry energy at saturation. Additional details and values of the model parameters can be found in Ref. [13].

The final state spectra of HIC are determined by making use of a minimum spanning-tree coalescence algorithm. It is applied to identify all clusters with $\mathrm{A} \leq 15$. A number of 23 additional clusters with $\mathrm{A}>15$ that correspond to known stable or unstable isotopes of $B, C, N$ and $O$ are also identified. Clusters with lifetimes larger than $1 \mathrm{~ms}$ are considered as stable while the rest decay until clusters stable against strong interaction decays are reached (ex. ${ }^{4} \mathrm{Li} \rightarrow \mathrm{p}+{ }^{3} \mathrm{He}$ ). For clusters with $\mathrm{A} \leq 15$ that do not correspond to a known stable or unstable isotope, protons or neutrons are evaporated for proton and neutron rich clusters respectively until a known nucleus is reached (ex. $1 p 8 n \rightarrow 6 n+{ }^{3} H$ ). Nucleons belonging to clusters with other masses are discarded from final spectra.

The values of the $r$-space coalescence parameters are determined from a fit of cluster and free nucleon multiplicities to experimental data. For the case of $\mathrm{Au}+\mathrm{Au}$ collisions at an impact energy of $400 \mathrm{MeV} /$ nucleon the FOPI cluster multiplicity experimental data for central collisions $(b \leq 2.0 \mathrm{fm})$ have been used [19], namely the multiplicities of $p, n,{ }^{2} \mathrm{H},{ }^{3} \mathrm{H},{ }^{3} \mathrm{He},{ }^{4} \mathrm{He}, \mathrm{Li}, \mathrm{Be}$ and C. The left panel of figure 1 presents the result of the fit for three values of the momentum space coalescence parameter $\delta p$. The experimental deuteron, $\mathrm{Be}, \mathrm{B}$ and $\mathrm{C}$ multiplicities are closely reproduced, in opposition to 

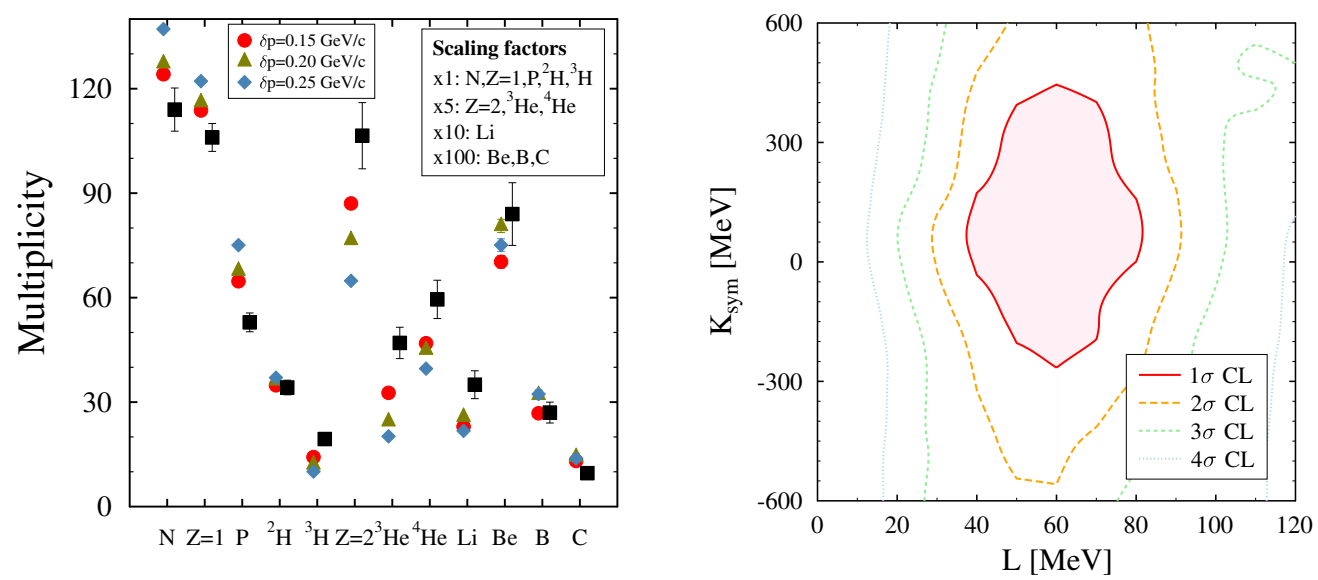

Figure 1. (Left panel) Multiplicities of free nucleons and clusters with $Z \leq 6$ for three values of the momentum space coalescence parameter compared to the experimental FOPI data [19] (full squares with error bars). The asy-EoS stiffness parameters have been set to $L=60.5 \mathrm{MeV}$ and $K_{\text {sym }}=-81.0 \mathrm{MeV}$. The values of the $r$-space coalescence parameters for each case are listed in Ref. [13]. (Right panel) Constraint for the slope $L$ and curvature $K_{s y m}$ extracted from FOPI-LAND npEFR and ASYEOS $p_{T}$ dependent nchEFR. Curves for the 1, 2, 3 and $4 \sigma$ confidence levels are shown.

similar models reported in the literature [7]. The multiplicities of Li, He isotopes and triton are underpredicted, but the discrepancy with respect to experimental values is smaller as compared to results reported elsewhere. The reason behind this improvement has been identified to be the momentum dependence of the MDI2 interaction.

\section{Selected results}

By keeping the value of the SE at saturation and its slope $L$ fixed the sensitivity of npEFR, nhEFR, nchEFR observables to the curvature $K_{\text {sym }}$ parameters can be investigated. It was found [13] that npEFR and nhEFR (or alternatively nchEFR) show different sensitivity to variations of $K_{\text {sym }}$. This is a direct consequence of the fact that these observables probe, on average, different density regions [9]. The combination of observables FOPI-LAND npEFR and ASYEOS nchEFR presents the best opportunity for constraining both $L$ and $K_{s y m}$ in view of the higher accuracy of the latter data set. The extracted values for the slope and curvature parameters read $L=59 \pm 24 \mathrm{MeV}$ and $K_{\text {sym }}=88 \pm 372 \mathrm{MeV}$ at $1 \sigma \mathrm{CL}$. The 1, 2, 3 and $4 \sigma \mathrm{CL}$ contour curves are plotted in the right panel of figure 1 . The minimum value for the quality-of-fit parameter is for the presented case $\chi_{\min }^{2} /$ point $=0.14$.

The extracted constraints are systemically affected by the under-prediction, by the model, of experimental cluster-to-proton multiplicity ratios. Consequently, the value of charged-particles elliptic flow $v_{2}^{c h}$ entering the expression of nchEFR needs to be corrected by a factor that has been estimated to lie in the interval $1.05 \leq f_{\text {corr }} \leq 1.15$. This correction leads to a softer value for $K_{\text {sym }}$. Additionally, the effect of poorer known model ingredients such as the values for the compressibility modulus of symmetric nuclear matter and isovector neutron-proton effective mass difference, scenarios used for in-medium nucleon-nucleon cross-sections or total energy conservation of the system and employed Pauli blocking algorithm on the extracted SE constraints has also been investigated. Their impact is summed up in quadrature to determine the theoretical uncertainty of the extracted constraint. The 
final result, obtained by using the more realistic value $f_{\text {corr }}=1.10$, is

$$
\begin{aligned}
L & =59 \pm 24(\exp ) \pm 16(\text { th }) \pm 10(\text { sys }) \mathrm{MeV} \\
K_{\text {sym }} & =0 \pm 370(\exp ) \pm 220(\text { th }) \pm 150(\text { sys }) \mathrm{MeV} .
\end{aligned}
$$

It is possible to extract a more precise constraint for the slope $L$, free of systematical uncertainties, from the FOPI-LAND npEFR data alone by taking into account the sensitivity of this observable to $K_{\text {sym }}$. By using a conservative range for the location of the real value of this parameter, $-300 \leq K_{\text {sym }} \leq$ $300 \mathrm{MeV}$, the uncertainty it induces on the extracted value of $L$ can be estimated. The extracted constraint for $L$ reads

$$
L=63 \pm 18(\exp ) \pm 14(\text { th }) \mathrm{MeV}
$$

Differences with respect to a previous study [10] were found to be equally due to a more accurate description of nucleon density profile of nuclei and usage of a coalescence algorithm rather than a density cut-off method to determine final spectra in the present study.

In conclusion, it has been shown that existing experimental data for neutron-to-proton and neutronto-charged particles elliptic flow ratios allow the extraction of constraints for both the slope and curvature of the symmetry energy at saturation. For the former parameter the result is compatible with constraints extracted from nuclear structure and astrophysical experimental data and of similar accuracy. The accuracy of the latter parameter is rather poor but it is nevertheless compatible with a stiff symmetry energy at densities equal or larger than twice the saturation density hinting for a possible solution of the hyperon puzzle.

\section{References}

[1] B.A. Li, L.W. Chen, C.M. Ko, Phys. Rept. 464, 113 (2008)

[2] J.M. Lattimer, M. Prakash, Phys. Rept. 442, 109 (2007)

[3] M. Baldo, G.F. Burgio, Prog. Part. Nucl. Phys. 91, 203 (2016)

[4] C. Drischler, A. Carbone, K. Hebeler, A. Schwenk, Phys. Rev. C94, 054307 (2016)

[5] I. Vidana, A. Polls, A. Ramos, L. Engvik, M. Hjorth-Jensen, Phys. Rev. C62, 035801 (2000)

[6] B.A. Li, Nucl. Phys. A708, 365 (2002)

[7] P. Russotto et al., Phys. Lett. B697, 471 (2011)

[8] M.D. Cozma, Phys. Lett. B700, 139 (2011)

[9] P. Russotto et al., Phys. Rev. C94, 034608 (2016)

[10] M.D. Cozma, Y. Leifels, W. Trautmann, Q. Li, P. Russotto, Phys. Rev. C88, 044912 (2013)

[11] Y. Wang, C. Guo et al., Phys. Rev. C89, 044603 (2014)

[12] Y. Leifels et al. (FOPI), Phys. Rev. Lett. 71, 963 (1993)

[13] M.D. Cozma (2017), arXiv: 1706.01300

[14] M.D. Cozma, Phys. Lett. B753, 166 (2016)

[15] M.D. Cozma, Phys. Rev. C95, 014601 (2017)

[16] D.T. Khoa, N. Ohtsuka, M.A. Matin, R.K. Puri, Nucl. Phys. A548, 102 (1992)

[17] V.S. Uma Maheswari, C. Fuchs, A. Faessler, L. Sehn, D.S. Kosov, Z. Wang, Nucl. Phys. A628, 669 (1998)

[18] C.B. Das, S.D. Gupta, C. Gale, B.A. Li, Phys. Rev. C67, 034611 (2003)

[19] W. Reisdorf et al. (FOPI), Nucl. Phys. A848, 366 (2010) 